\title{
Grupos de Pesquisa de Educação em Enfermagem do Brasil*
}

\author{
NURSING EDUCATION RESEARCH GROUPS IN BRAZIL
}

GRUPOS DE INVESTIGACIÓN DE EDUCACIÓN EN ENFERMERÍA DE BRASIL

\author{
Vânia Marli Schubert Backes', Marta Lenise do Prado², Mônica Motta Lino³, Fabiane Ferraz ${ }^{4}$, \\ Kenya Schmidt Reibnitz ${ }^{5}$, Bruna Pedroso Canever ${ }^{6}$
}

\begin{abstract}
RESUMO
O estudo tem por objetivo caracterizar os Grupos de Pesquisa em Educação em Enfermagem do Brasil quanto à sua organização. Pesquisa documental, descritiva, quantitativa. As informações foram coletadas no Banco de Dados e Estatísticas do Portal Online do CNPq - censo 2006. O Brasil possui 47 Grupos de Pesquisa em Educação em Enfermagem, com 412 pesquisadores, dos quais $91 \%$ apresentam título de mestrado, doutorado ou pós-doutorado. Dos 307 estudantes, 92\% são graduandos de Enfermagem, porém apenas $9 \%$ são bolsistas de iniciação científica. Entre os 112 técnicos, $75 \%$ são de Enfermagem, 46\% possuem titulação de mestre ou doutor. Há um número expressivo de Grupos que contribuem significativamente para a produção de conhecimento no setor de educação, em nível latino-americano. Todavia, ainda são muitos os desafios a serem superados como a frágil interdisciplinaridade, a limitada integração ensino-serviço, o baixo fomento de bolsas de iniciação científica e as significativas desigualdades no acesso e desenvolvimento de pesquisas nas diferentes regiões do país.
\end{abstract}

\section{DESCRITORES}

Grupos de pesquisa

Educação em enfermagem

Pesquisa em educação de enfermagem

\begin{abstract}
The aim of this study is to characterize the organization of nursing education research groups in Brazil. This is a quantitative, descriptive and documentary study. Census data for 2006 were collected from the CNPq database website. Brazil has 47 education research groups, comprised of 412 researchers, of whom $91 \%$ have masters, doctoral or postdoctoral degrees. There are 307 students, of whom $92 \%$ are nursing undergraduates. However, only $9 \%$ are recipients of young investigator grants. There are also 112 technicians, of whom $75 \%$ are nursing professionals; $46 \%$ have a masters or doctoral degree. In Brazil, there are a considerable number of nursing education research groups, which contribute significantly to scientific production of nursing knowledge in Latin America. In this scenario, there are many challenges to be overcome: poor interdisciplinary cooperation; limited integration between education and practice; low number of grants awarded to young investigators and significant inequalities between the country's geographic regions in terms of access to research development.
\end{abstract}

\section{DESCRIPTORS \\ Research groups \\ Education, nursing \\ Nursing education research}

\begin{abstract}
RESUMEN
Estudio que objetiva caracterizar los Grupos de Investigación de Educación en Enfermería de Brasil respecto de su organización. Investigación documental, descriptiva, cuantitativa. Informaciones recolectadas en Banco de Datos y Estadísticas, Portal Online del CNPq-censo 2006. Brasil posee 47 Grupos de Investigación de Educación en Enfermería, con 412 investigadores, de los que 91\% ostenta títulos de maestría, doctorado o post-doctorado. De los 307 estudiantes, $92 \%$ es alumno de Enfermería, sin embargo apenas $9 \%$ es becado para iniciación científica. Entre los 112 técnicos, $75 \%$ es de Enfermería, $46 \%$ poseen título de maestría o doctorado. Existe un número importante de Grupos que contribuye significativamente en producción de conocimiento del sector educativo, a nivel latinoamericano. Igualmente, hay muchos desafíos a superar, como la frágil interdisciplinaridad, la limitada integración enseñanza-servicio, el bajo fomento de becas de iniciación científica y las significativas desigualdades en acceso y desarrollo de investigaciones en las diferentes regiones geográficas del país.

\section{DESCRIPTORES \\ Grupos de investigación \\ Educación en enfermería \\ Investigación en educación de enfermería}

\footnotetext{
* Extraído do Projeto de Pesquisa "A produção investigativa de educação em enfermagem no Brasil: o estado da arte", Universidade Federal de Santa Catarina, 2006. 'Doutora em Enfermagem. Docente do Departamento de Enfermagem e do Programa de Pós-Graduação em Enfermagem da Universidade Federal de Santa Catarina. Líder do Grupo de Pesquisa em Educação em Enfermagem e Saúde. Pesquisadora do Conselho Nacional de Desenvolvimento Científico e Tecnológico - CNPq. Florianópolis, SC, Brasil. oivania@ccs.ufsc.br ${ }^{2}$ Doutora em Enfermagem. Docente do Departamento de Enfermagem e do Programa de Pós-Graduação em Enfermagem da Universidade Federal de Santa Catarina. Vice-Líder do Grupo de Pesquisa em Educação em Enfermagem e Saúde. Pesquisadora do CNPq. Florianópolis, SC, Brasil. mpradop@ccs.ufsc.br ${ }^{3}$ Mestre em Enfermagem. Doutoranda do Programa de Pós-Graduação em Enfermagem da Universidade Federal de Santa Catarina. Bolsista da Coordenação de Aperfeiçoamento de Pessoal de Nível Superior - CAPES. Membro do Grupo de Pesquisas em Educação e Saúde. Florianópolis, SC, Brasil. cmonicafloripa@hotmail.com ${ }^{4}$ Doutora em Enfermagem. Membro do Grupo de Pesquisa em Educação em Enfermagem e Saúde. Criciúma, SC, Brasil. olaferraz@yahoo.com.br ${ }^{5}$ Doutora em Enfermagem. Docente do Departamento de Enfermagem e do Programa de Pós-Graduação em Enfermagem da Universidade Federal de Santa Catarina. Coordenadora do Centro de Ciências da Saúde da Universidade Federal de Santa Catarina. Membro do Grupo de Pesquisas em Educação em Enfermagem e Saúde. Florianópolis, SC, Brasil. kenya@ccs. ufsc.br ${ }^{6}$ Enfermeira. Mestre em Enfermagem. Doutoranda em Enfermagem do Programa de Pós-Graduação em Enfermagem da Universidade Federal de Santa Catarina. Bolsista do CNPq. Membro do Grupo de Pesquisa em Educação em Enfermagem e Saúde. Florianópolis, SC, Brasil. olabruna@gmail.com
} 


\section{INTRODUÇÃO}

No cenário mundial, a pesquisa em Enfermagem vem apresentando importantes avanços, ainda que com grandes diferenças entre países e continentes, em especial, no tocante à pesquisa do setor de Educação e Enfermagem ${ }^{(1)}$. Neste contexto, o panorama de Ciência e Tecnologia em Enfermagem na América Latina vem sendo fortalecido por meio de organismos estatais criados para este fim, com a intenção de incentivar e alavancar o setor de produção, disseminação e transferência de novos conhecimentos, sendo que a liderança brasileira no cenário latino-americano é reconhecida ${ }^{(2-4)}$.

O Brasil, a Venezuela e a Colômbia são identificados como países latino-americanos com maior trajetória nesta caminhada, tendo em vista a organização da investigação por meio de Grupos de Pesquisa, linhas de pesquisa e centros de investigação, assim como a constituição de redes de informação disponibilizadas na internet. Esta produção, por sua vez, tem relação direta com a formação universitária, em especial com os Programas de Pós-Graduação Stricto Sensu, que favorecem a formação de pesquisadores críticoreflexivos e qualificados para produzir novos conhecimentos $^{(2,5)}$.

A área da Enfermagem vem repensando seus modos de fazer, de pesquisar e de educar, o que reflete avanços e mudanças no desenvolvimento curricular dos cursos de formação profissional, ou seja, do ensino de Graduação e Pós-Graduação. As novas tendências e inovações pedagógicas produzidas dentro da academia, atreladas ao desenvolvimento científico e tecnológico realizados nos Grupos de Pesquisa, têm contribuído e também têm sido produto dos diferentes processos de produção científica no setor de Educação em Enfermagem do Brasil.

Historicamente, a área de Enfermagem apresenta uma predominância de práticas não profissionais. A discussão sobre a chamada explosão educativa e científica da Enfermagem nos últimos 20 anos na América Latina tem apontado avanços para o fortalecimento e a compreensão da Enfermagem como força de trabalho e produtora de conhecimentos ${ }^{(4)}$, a exemplo de outros países, com destaque, aos da América Anglo-saxônica. Considerando que a educação deve estar intrínseca ao processo de trabalho e que este processo de racionalização do saber é condição precedente à profissionalização de qualquer atividade humana, acredita-se que o fortalecimento da Enfermagem pode ocorrer por meio da evolução da ciência e da educação, logo, é imprescindível que pesquisadores e educadores de Enfermagem latino-americanos potencializem o desenvolvimento da ciência e da educação em Enfermagem, tendo em vista as demandas sociais atendidas pelo setor ${ }^{(2)}$.
Os pesquisadores de Enfermagem brasileiros organizaram os Grupos de Pesquisas a partir das orientações do Conselho Nacional de Desenvolvimento Científico e Tecnológico (CNPq) para impulsionar o processo de desenvolvimento científico para a gestão da produção de conhecimentos e a consolidação de sua base científica. Cabe salientar que Grupo de Pesquisa (GP), no Diretório dos Grupos de Pesquisas no Brasil do CNPq, pode ser definido como

um conjunto de indivíduos organizados hierarquicamente, cujo fundamento organizador da hierarquia é a experiência, o destaque e a liderança no terreno científico ou tecnológico, em que há envolvimento profissional e permanente com atividades de pesquisa, no qual o trabalho se organiza em torno de linhas comuns de pesquisa e que, em algum grau compartilha instalações e equipamentos ${ }^{(6)}$.

Desse modo, na área de Enfermagem, mais especificamente no setor de Educação em Enfermagem no Brasil, os Grupos de Pesquisa em Educação em Enfermagem (GPEE) vêm atuando como estruturas parceiras junto aos Programas de Pós-Graduação em Enfermagem (PPGE), à comunidade e serviços de saúde, a fim de proporcionar uma formação profissional voltada à realidade social, à construção e incentivo de políticas de desenvolvimento profissional da área e ao despertar para uma pedagogia crítica por meio do enfrentamento de problemas complexos do quotidiano ${ }^{(7)}$.

Os Programas de Pós-Graduação em Enfermagem no Brasil vêm se desenvolvendo amplamente no decorrer das últimas décadas. Produção Científica e Pós-Graduação são co-dependentes no processo de desenvolvimento da profissão, visto que uma é fundamental para que a outra atenda às demandas da sociedade ${ }^{(4)}$. Enquanto a PósGraduação incentiva e direciona as produções, por meio de Grupos de Pesquisa, os produtos, muito mais que apenas números, são dispositivos que evidenciam avanços e retrocessos no processo de construção do conhecimento de uma disciplina. Assim, os Grupos de Pesquisa vêm desempenhando fundamental papel na construção de novas abordagens teórico-metodológicas, contribuindo para a formação e qualificação de pesquisadores que investem em produção e divulgação de conhecimento científico e para o processo de captação de investimentos oriundos de agências de fomento à pesquisa. Em outros aspectos, o desenvolvimento crescente e constante dos Grupos de Pesquisa tem ampliado a orientação e abrangência da produção do conhecimento ${ }^{(8)}$.

Neste contexto, cabe ressaltar que os Grupos de Pesquisa, em especial aqueles que trabalham a temática Educação em Enfermagem, são de extrema relevância para o processo de desenvolvimento da Enfermagem brasileira porque realizam pesquisas que qualificam as discussões sobre a formação profissional, a criação e 
aplicação de novas tecnologias educacionais, os processos de educação permanente e continuada em saúde, a assistência em saúde, a construção e investigação acerca de políticas, programas e projetos sociais, entre tantos outros fatores que influenciam e potencializam intervenções críticas dos profissionais ${ }^{(7,9-11)}$.

Frente a este relevante panorama, o presente estudo tem como objetivo caracterizar os Grupos de Pesquisa de Educação em Enfermagem (GPEE) do Brasil quanto à sua organização, dada a importância de conhecer esta realidade para superar fragilidades e estimular a formulação de políticas de desenvolvimento e fortalecimento dos grupos de pesquisa neste âmbito.

\section{MÉTODO}

Pesquisa do tipo documental, descritiva, quantitativa. Os dados foram coletados a partir do censo de 2006, disponível no Banco de Dados e Estatísticas do Portal on-line do $\mathrm{CNPq}^{(6)}$, que corresponde especificamente às informações disponibilizadas pelos Grupos de Pesquisa referentes aos anos de 2005 e 2006.

Foram selecionadas as seguintes variáveis: Área de Atuação, por UF e por Instituição. Na sequência, foi realizado o filtro da primeira variável, sendo escolhida a área Enfermagem. Assim, o sistema gerou uma tabela contendo todos os Grupos de Pesquisa em Enfermagem (GPE) do Brasil em 2006. Foram escolhidos os Grupos de Pesquisa em Educação em Enfermagem brasileiros a partir da apresentação das palavras educação, ensino ou formação no nome do grupo.

Para facilitar a organização dos dados, foram construídas planilhas no Microsoft Excel ${ }^{\circledR} 2003$ divididas por região geográfica do Brasil, sendo identificados os seguintes elementos:

a) o número de Bolsistas de Iniciação Científica entre os estudantes de graduação;

b) o número total de Grupos de Pesquisa na área de Enfermagem;

c) o número específico de Grupos de Pesquisa de Educação em Enfermagem, cujo critério para identificação foi apresentar a palavra educação ou sinônimos (ensino e formação) no nome do grupo;

d) a presença de Programas de Pós-Graduação Stricto Sensu em Enfermagem;

e) o nome completo, o ano de início e a sigla do Grupo de Pesquisa de Educação em Enfermagem;

f) o número, a formação, a titulação e a atuação profissional dos pesquisadores dos Grupos de Pesquisa de Educação em Enfermagem; g) o número, a formação e a titulação dos estudantes dos Grupos de Pesquisa de Educação em Enfermagem;

h) as instituições e o seu caráter institucional;

i) o número, a formação, a titulação e a atuação profissional dos técnicos dos Grupos de Pesquisa de Educação em Enfermagem;

j) o número de Bolsistas de Apoio Técnico entre os técnicos;

k) o número de pesquisadores, estudantes e técnicos com titulação na área de Educação dos Grupos de Pesquisa de Educação em Enfermagem.

Os dados sobre os grupos de pesquisa, os nomes dos pesquisadores, estudantes e técnicos foram complementados por meio das informações disponibilizadas online no Diretório dos Grupos de Pesquisa do CNPq. Em relação à formação, à titulação, à titulação na área de educação e à atuação profissional, os dados foram coletados e organizados em tabelas utilizando o programa Microsoft Word $2003^{\circ}$ a partir da consulta a todos os Currículos Lattes - CNPq dos pesquisadores, estudantes e técnicos que surgiram na busca realizada no Diretório dos Grupos. Os dados sobre os Programas de Pós-Graduação Stricto Sensu na Enfermagem foram coletados no site da Coordenação de Aperfeiçoamento de Pessoal de Nível Superior (CAPES) - Brasil.

Como se trata de uma pesquisa documental, cujo conteúdo disponibilizado é de caráter público, este estudo não foi submetido ao Comitê de Ética em Pesquisa com Seres Humanos. No entanto, cabe ressaltar que os pesquisadores seguiram todos os preceitos éticos necessários para a análise e divulgação dos dados da pesquisa dessa natureza.

\section{RESULTADOS}

No ano de 2006, os Cursos de Graduação em Enfermagem do Brasil encontravam-se distribuídos em 80 Instituições de Ensino Superior (IES) Públicas, e em 388 Instituições de Ensino Superior (IES) Privadas. Identificou-se, a partir do censo 2006 publicado pelo CNPq, que o país apresentava 331 Grupos de Pesquisa em Enfermagem, distribuídos em 56 (IES Públicas) e 25 (IES Privadas) com Curso de Graduação em Enfermagem.

O número total de Grupos de Pesquisa de Educação em Enfermagem (GPEE) no Brasil, ou seja, que apresentaram, conforme elucidado na metodologia, a palavra educação, ensino ou formação no nome do Grupo foram 47, distribuídos em 23 (IES Públicas) e 10 em (IES Privadas), conforme apresentado na Figura 1. Levandose em conta o número total de Grupos de Pesquisa em Enfermagem (331), percebe-se que 14\% trabalham com o tema educação em todo o país. 


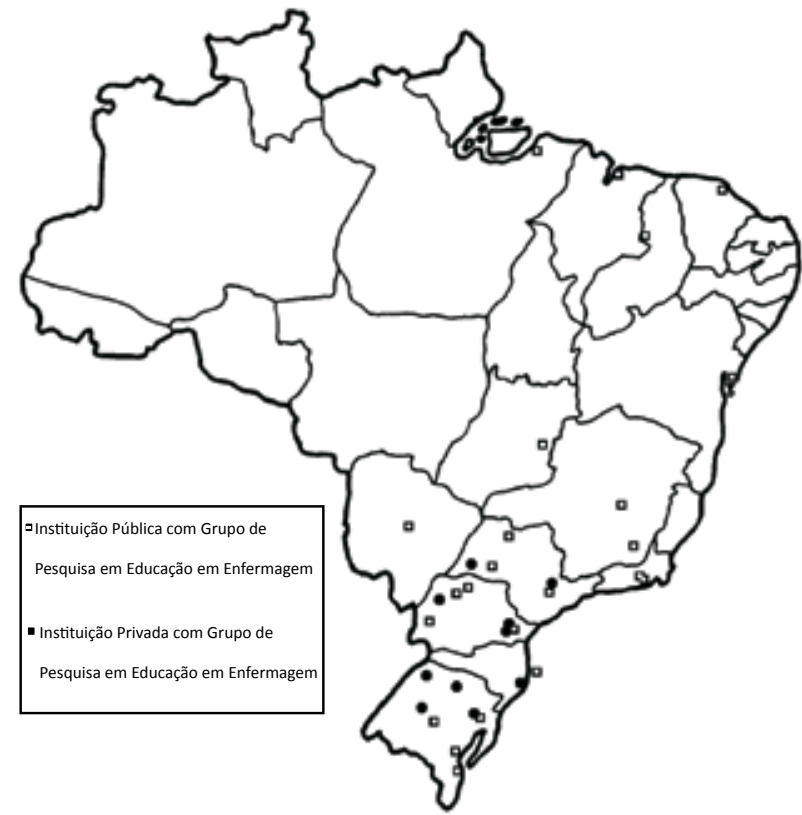

Figura 1 - Instituições de Ensino Superior (Públicas e Privadas) que possuem Grupos de Pesquisa de Educação em Enfermagem no Brasil - CNPq/Brasil, 2005-2006
Considerando-se o total de IES com Cursos de Graduação em Enfermagem no Brasil em 2006, nota-se que $28,7 \%$ das IES Pública apresentam Grupos de Pesquisa de Educação em Enfermagem, contra apenas 2,6\% das IES Privadas. Em relação ao ano de criação dos GPEE, dois se constituíram na década de 80,15 se estruturaram na década de 90 do século XX e 30 a partir do ano 2000.

Entre as IES que apresentam Grupos de Pesquisa de Educação em Enfermagem, dezessete possuem Programa de Pós-Graduação Stricto Sensu em Enfermagem reconhecidos pela Coordenação de Aperfeiçoamento de Pessoal de Nível Superior (CAPES), sendo uma de caráter privado e dezesseis de caráter público. Entre os 25 Programas de Pós-Graduação Stricto Sensu em Enfermagem do Brasil até 2006, sete se estruturaram na década de 70 , três na década de 80 , seis se constituíram na década de 90 do século XX e nove a partir do ano 2000.

A Figura 2 apresenta a distribuição dos Grupos de Pesquisa em Enfermagem (GPE), dos Grupos de Pesquisa em Educação em Enfermagem (GPEE) e dos respectivos Pesquisadores (P), Estudantes (E) e Técnicos (T) da área de Enfermagem nos GPEE discriminados por região em todo território brasileiro.

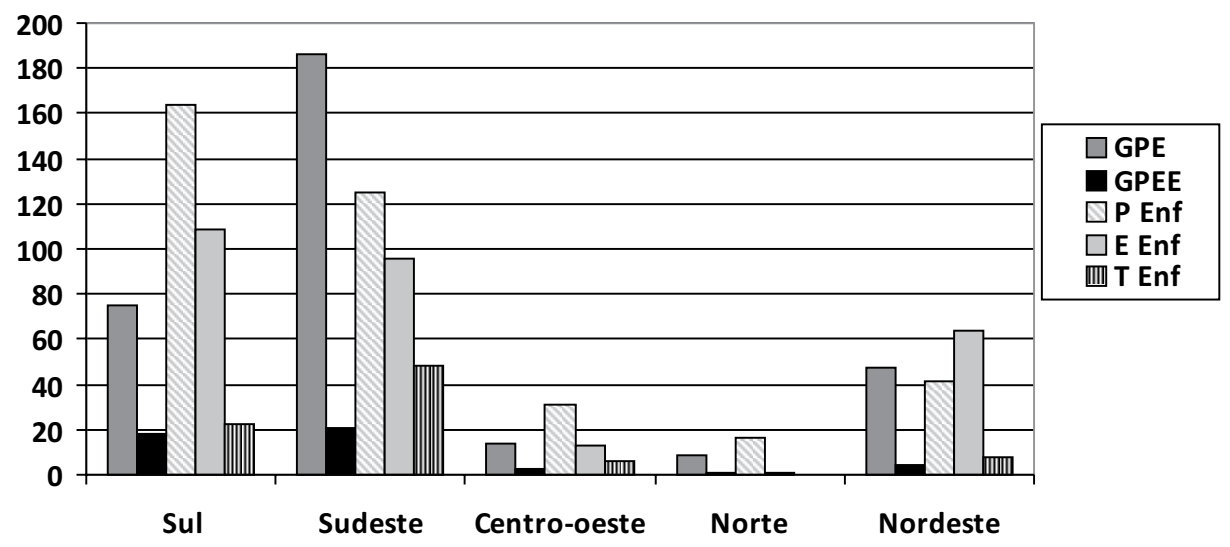

Figura 2 - Distribuição dos Grupos de Pesquisa segundo regiões geográficas do Brasil - CNPq/Brasil, 2005-2006

As Regiões Sul e Sudeste do Brasil possuem 79\% dos Grupos de Pesquisa em Enfermagem, bem como 83\% dos Grupos de Pesquisa em Educação em Enfermagem existentes no país no período do estudo. Consequentemente apresentam um número majoritário de pesquisadores, estudantes e técnicos na área de Enfermagem em relação às demais regiões do país.

A Figura 3 apresenta o número total de pesquisadores, Estudantes e Técnicos dos Grupos de Pesquisa em Enfermagem (GPE) e Grupos de Pesquisa de Educação em Enfermagem (GPEE) do Brasil em 2006.

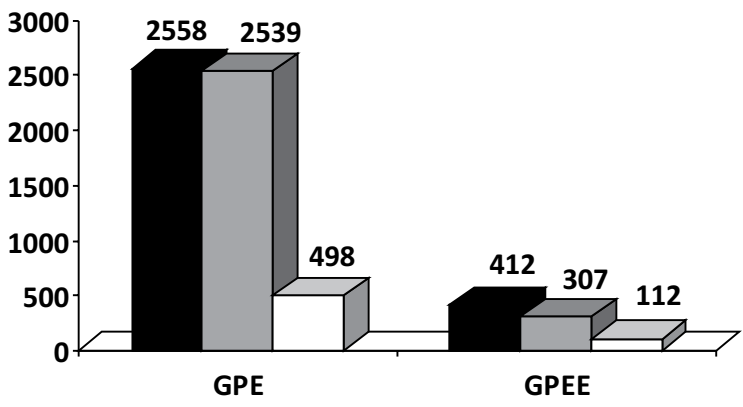

Pesquisadores $\square$ Estudantes $\square$ Técnicos

Figura 3 - Número total de Pesquisadores - CNPq/Brasil, 2005-2006 
A Figura 3 indica que $16 \%$ dos Pesquisadores, $12 \%$ dos Estudantes e $22 \%$ dos Técnicos que atuam em Grupos de Pesquisa de Enfermagem no Brasil dedicam-se a estudar a temática de Educação em Enfermagem.

Convém destacar que, a partir de consulta ao Currículo Lattes/CNPq, entre os Pesquisadores dos GPEE, 377 são da área de Enfermagem e apenas 33 são de outras áreas do conhecimento, como Psicologia, Fisioterapia, Terapia Ocupacional, Educação Física, Medicina, Serviço Social, Direito, Odontologia, Engenharia de Produção, Farmácia, Administração, Matemática, Ciências Biológicas e Pedagogia, e dois não possuíam Currículo Lattes. Ainda, é possível constatar que $66 \%$ dos pesquisadores atuam na docência, $5 \%$ atuam exclusivamente na assistência, $28 \%$ trabalham na docência e assistência e $1 \%$ não informou.

Com relação aos Estudantes dos GPEE, 283 são de Enfermagem, 18 são de outras áreas do conhecimento, a saber: Psicologia, Ciência Sociais, Fisioterapia, Terapia Ocupacional, Educação Física, Medicina, Comunicação Social, Ciências da Educação, Odontologia, Nutrição, Ciências Contábeis, e seis não possuíam Currículo Lattes.

Sobre os Técnicos dos GPEE, 84 são da área de Enfermagem, 17 de outras áreas de conhecimento, como: Informática, Letras, História, Nutrição, Publicidade e Propaganda, Serviço Social, Psicologia, Medicina e 11 não possuíam Currículo Lattes. Em relação à atuação profissional dos técnicos, 20 trabalham na docência, 21 na assistência, 29 na docência e assistência, 39 não informaram.

A titulação dos membros dos GPEE é apresentada na Tabela 1.

Tabela 1 - Distribuição dos Pesquisadores, Estudantes e Técnicos participantes dos Grupos de Pesquisa de Educação em Enfermagem do Brasil, segundo a titulação - CNPq/Brasil, 2005-2006

\begin{tabular}{lccc}
\hline Titulação & Pesquisadores & Estudantes & Técnicos \\
\hline Pós-doutorado & 13 & 00 & 00 \\
Doutorado & 215 & 46 & 20 \\
Mestrado & 148 & 68 & 32 \\
Especialização & 28 & 12 & 28 \\
Graduação & 6 & 180 & 27 \\
Ensino Médio & 0 & 0 & 5 \\
Não Informaram & 2 & 1 & 0 \\
Total & 412 & 307 & 112 \\
\hline
\end{tabular}

Fonte: Censo 2006 - CNPq e Plataforma Lattes/CNPq

Verifica-se, portanto, que os Grupos de Pesquisa de Educação em Enfermagem do Brasil possuem um contingente de $91 \%$ do seu quadro de pesquisadores com mestrado, doutorado e pós-doutorado. Entre o total de pesquisadores apresentados, 159 possuem, entre suas titulações, uma titulação na área da Educação, sendo nove doutores em educação, 48 mestres em educação e 102 especialistas na área. Em relação aos Técnicos, 52 possuem titulação em nível stricto sensu, 28 possuem titulação latu sensu, 27 graduação e 39 não informaram.

A análise dos Currículos Lattes dos estudantes de graduação apontou que 28 , ou seja, $9 \%$ são bolsistas de Iniciação Científica, os demais apresentam um caráter de participação voluntária nos GPEE. Em relação aos Técnicos, três são bolsistas de Apoio Técnico.

\section{DISCUSSÃO}

Os dados apresentados na Figura 1 corroboram com o cenário latino-americano, visto que a maior concentração de GPEE encontra-se em IES públicas. Ainda, é possível perceber que, na Enfermagem brasileira, o início da formação dos Grupos ocorreu na década de 80, paralelamente ao crescimento dos Programas de Pós-Graduação, porém, a consolidação desses grupos aconteceu a partir da organização e sistematização efetuada pelo $\mathrm{CNPq}^{(10)}$. Logo, elucida -se a importância dos Programas de Pós-Graduação no fomento à criação de Grupo de Pesquisa na Enfermagem e o papel do CNPq para consolidação desses grupos.

O contingente de GPEE em relação ao número total de GP em Enfermagem no Brasil demonstra um potencial de produção, transferência e disseminação de conhecimentos relativos à formação de recursos humanos em Enfermagem, cuja produção pode contribuir para a indução de políticas educativas e proposição de novas metodologias. Portanto, os processos educativos em Enfermagem podem ser uma possibilidade de compreender melhor o processo de ensino, bem como a sua importância no desenvolvimento de ciência e tecnologia.

No entanto, os dados apresentados na Figura 2 evidenciam as desigualdades e desequilíbrios intrarregionais, visto que as Regiões Sul e Sudeste destacam-se por possuir o maior número de Grupos de Pesquisa, tanto de Enfermagem, quanto de Educação em Enfermagem, o que referenda a consolidação de um potencial de cientistas, de produção e de infraestrutura assimétrica em relação às demais regiões do Brasil, aspecto que se destaca não apenas no tocante à produção de conhecimento, mas em dimensões socioeconômicas e culturais neste país de dimensões continentais. Esta realidade demonstra a necessidade de adoção de políticas indutoras que favoreçam o desenvolvimento e equilibrem essas desigualdades que conduzem a iniquidades regionais, haja vista a desigualdade no que diz respeito à disponibilidade de programas de pós-graduação e à quantidade de pessoal qualificado como mestres e doutores, principalmente nas Regiões Norte e Centro-Oeste do Brasil(12).

Atualmente, no Brasil, pode-se considerar que o maior desafio no que concerne às escolas de formação de mestres e doutores em Enfermagem é a exigência de que os enfermeiros exerçam a função docente em nível de graduação e pós-graduação ao mesmo tempo em que administram a investigação científica e a extensão, sem desconsiderar as de- 
mandas de produtividade acadêmica no qual o sistema de professorado vem se fundamentando.

Desse modo, a produção de novos conhecimentos e seus reflexos na estrutura constitutiva da força de trabalho em Enfermagem acelera e qualifica o processo de profissionalização da área, sendo que os temas mais desafiadores permeiam o âmbito pedagógico, político e econômico ${ }^{(13)}$. Estes desafios encontram-se integrados em uma atualidade complexa, que necessita da promoção de alianças e agendas estratégicas conjuntas para a planificação do processo de educação e do desenvolvimento de ciência e tecnologia em Enfermagem no Brasil e na América Latina, de modo superar a fragilidade da integração ensino-serviço dentro dos GPEE, manifestada pela prevalência de pesquisadores que atuam exclusivamente na docência(7).

Outro destaque deste estudo é a pequena, no entanto, importante participação de pesquisadores de outras áreas nos GPEE. As novas fronteiras com que a ciência se depara indicam que o conhecimento específico não é suficiente para entender e atender a complexidade dos fenômenos estudados. Assim, torna-se indispensável abrir as disciplinas umas às outras para enriquecê-las com os pontos de vista provenientes de outros horizontes. Esse processo gera a possibilidade de associar conhecimento como alternativa para a superação dos limites que a própria ciência criou no entendimento especializado de seus objetos ${ }^{(14)}$.

Se, por um lado, a interdisciplinaridade nos GPEE ainda é frágil, por outro, a busca por fundamentação na área de Educação pelos próprios profissionais de Enfermagem pode ser um indicativo da procura por cursos de pós-graduação que despertaram interesse e estão disponíveis nas regiões dos pesquisadores, mesmo não sendo na área de Enfermagem. $O$ fato de $38,6 \%$ de pesquisadores apresentarem uma titulação específica na área da educação confere maior sustentação teórica aos propósitos do ensino, pesquisa e extensão. Ao mesmo tempo, a escolha pela área de Educação demonstra comprometimento e necessidade de ampliar o conhecimento para efetivar o avanço científico neste campo em interface com a Enfermagem.

Todavia a interdisciplinaridade, ainda incipiente, elucidada pela pouca participação de profissionais de outras áreas do conhecimento e do serviço, aparece como um desafio a ser superado nos GPEE. Isto porque a interdisciplinaridade significa o envolvimento de diferentes áreas do conhecimento, pesquisas e abordagens multidisciplinares, por meio das quais é possível articular diferentes olhares, que podem contribuir para a compreensão e solução de problemas e produção de conhecimentos pertinentes ${ }^{(15-16)}$.

Além disso, estudos interdisciplinares constituem-se em um processo de responder questões, resolver problemas ou direcionar tópicos tão amplos ou complexos que a abordagem disciplinar não é suficiente para a sua compreensão ou solução, promovendo a combinação de conhecimento de diferentes especialidades, de forma a dar novos enfoques a um problema real ${ }^{(17)}$. Essa combinação de disciplinas adiciona valor ao processo, sendo possível perceber que o resultado obtido pelo estudo conjunto é mais interessante do que a soma das contribuições individuais das partes.

Os achados em relação à titulação dos pesquisadores corroboram com o panorama brasileiro de destaque na formação de doutores em Enfermagem na América Latina, tendo em vista a soma de 20 anos de experiência nesta área em detrimento de outros países como Argentina, Colômbia, Chile, México e Venezuela, que iniciaram os cursos de doutorado no final do século XX e início do século $X X I^{(2,5)}$.

Contudo, outra fragilidade apontada nesse estudo diz respeito ao pequeno número de bolsistas de pesquisa, tanto em relação à Iniciação Científica, pois a percentagem de bolsistas em relação ao total de estudantes de graduação que participam dos GPEE é muito pequena, quanto aos bolsistas de Apoio Técnico, que se caracterizam em número ínfimo. Ainda que o número insuficiente de bolsas concedidas por agências de fomento à formação e fixação institucional de novos pesquisadores, em particular, para os alunos e técnicos não seja um problema exclusivo da área de Enfermagem, este se constitui num importante desafio a ser superado.

\section{CONCLUSÃO}

Considera-se que reconhecer o cenário da produção de conhecimento na Educação e Enfermagem brasileira e, em especial, suas limitações e desafios é relevante, visto que contribui para a reorientação de políticas e práticas, tanto no contexto micro como macropolítico e social. Por meio dessas caracterizações, torna-se possível estruturar políticas e práticas capazes de impulsionar a reconstrução e a consolidação de processos formativos em Enfermagem no Brasil que favoreçam a consolidação do sistema de saúde brasileiro e o fortalecimento da profissão enquanto disciplina que produz e consome ciência.

Neste panorama, a prática da construção de conhecimento interdisciplinar exige do pesquisador uma postura diferenciada daquela que tradicionalmente é adotada. A necessidade de desenvolver novos paradigmas e métodos, essenciais para assegurar o rigor e a confiabilidade do conhecimento gerado, exige a renúncia de paradigmas e métodos já consolidados nos diversos campos disciplinares, bem como a constituição de equipes verdadeiramente multidisciplinares, o que se configura como um desafio a ser superado pelos Grupos de Pesquisa em Educação em Enfermagem brasileiros. Do mesmo modo, outro aspecto que merece destaque trata-se da fragilidade da integração ensino-serviço dentro dos Grupos, manifestada pela prevalência de pesquisadores e técnicos que atuam exclusivamente na docência. 
Em síntese, a constituição de 47 Grupos de Pesquisa em Educação em Enfermagem no Brasil em 2006, pressupõe um número expressivo e uma consequente contribuição para a produção do conhecimento na América Latina. Porém, muitos desafios necessitam ser superados como a frágil interdisciplinaridade, a limitada integração ensino-serviço, o baixo fomento de bolsas para o avanço da área da Educação em Enfermagem e as significativas desigualdades no acesso e desenvolvimento de pesquisas nas diferentes regiões geográficas do país.

\section{REFERÊNCIAS}

1. Polit DF, Beck CT. International differences in nursing research, 2005-2006. J Nurs Scholarsh. 2009;41(1):44-53.

2. Malvárez SM, Castrillón-Agudelo MC. Panorama de la fuerza de trabajo en enfermería en América Latina. Washington: OPAS; 2005

3. Mendoza-Parra S, Paravic-Klijn T, Muñoz-Muñoz AM, Barriga OA, Jiménez-Contreras E. Visibility of Latin American nursing research (1959-2005). J Nurs Scholarsh. 2009; 41(1):54-63.

4. Silva MJP, Egry EY, Ângelo M, Barbosa MAM, Sousa RMC, Castilho V, et al. Nursing knowledge production: from the research idea to the publishing in a qualified journal. Rev Esc Enferm USP [Internet]. 2009 [cited 2010 May 15];43(n.esp 2):1347-51. Available from: htp://www.scielo.br/pdf/reeusp /v43nspe2/en_a35v43s2.pdf

5. Castrillón MC. Los nuevos programas de doctorado en Enfermería y su contribución en la reducción de la demanda de las drogas en América Latina: retos y perspectivas. Monterrey: UNANL/OEA/CICAD; 2003.

6. Brasil. Ministério de Ciências e Tecnologia; Conselho Nacional de Desenvolvimento Científico e Tecnológico (CNPq). Grupos de Pesquisa - Censos [Internet]. Brasília; 2008 [citado 2010 jun 15]. Disponível em: http://dgp.cnpq.br/censos/inf_gerais/ index_que_eh.htm

7. Backes VMS, Canever BP, Ferraz F, Lino MM, Prado ML, Reibnitz KS. Grupos de Pesquisa de Educação em Enfermagem da Região Sul do Brasil. Rev Gaúcha Enferm. 2009;30(2):249-56.

8. Lino MM. Produção científica dos Grupos de Pesquisa em Educação em Enfermagem da Região Sul do Brasil [dissertação]. Florianópolis: Programa de Pós-Graduação em Enfermagem, Universidade Federal de Santa Catarina; 2009.
Por fim, a limitação desse estudo encontra-se no fato de ser um recorte da realidade dos Grupos de Pesquisa em Educação em Enfermagem nos anos de 2005-2006. Assim, destaca-se a necessidade da realização de outros estudos sobre essa temática no Brasil, enfocando as linhas de investigação estruturadas, a produção científica desenvolvida nestes grupos, bem como a possibilidade de realizar um estudo semelhante ao aqui apresentando, com os dados do Censo de 2008 do CNPq, a fim de traçar um paralelo de avaliação do processo de desenvolvimento do setor de educação na área de Enfermagem.

9. Rodrigues RAP, Erdmann AL, Fernandes JD, Araújo TL. Pós-Graduação em Enfermagem no Brasil e no Nordeste. Rev Gaúcha de Enferm. 2007;28(1):70-8.

10. Servo MLS, Oliveira MAN. A pesquisa e o enfermeiro com qualidade formal e qualidade política: caminho para a consolidação da enfermagem como ciência. Sitientibus. 2005; 33(2): 11-21.

11. Reibnitz KS, Prado ML. Formação do profissional crítico-criativo: a investigação como atitude de (re)conhecimento do mundo. Texto Contexto Enferm. 2003;12(1):26-33.

12. Prado ML, Backes VMS, Santana, ME, Souza ML. Políticas públicas na formação em saúde: contribuição da enfermagem para superação das desigualdades regionais brasileiras. Texto Contexto Enferm. 2007;16(3):531-5.

13. Malvárez SM, Castrillón-Agudelo MC. Panorama de la fuerza de trabajo en enfermería en América Latina: segunda parte. Rev Enfermería. 2006;14(3):145-65.

14. Bianchi F. O caminho do método. In: Pena-Veiga A, Nascimento $E P$, editores. O pensar complexo: Edgar Morin e a crise da modernidade. 2a ed. Rio de Janeiro: Garamond; 1999. p. 119-28.

15. Meirelles BHS, Erdmann AL. A interdisciplinaridade como construção do conhecimento em saúde e enfermagem. Texto Contexto Enferm. 2005;14(3):411-8.

16. Roese A, Souza AC, Porto GB, Colomé ICS, Costa LED. A produção do conhecimento na enfermagem: desafios na busca de reconhecimento no campo interdisciplinar. Rev Gaúcha Enferm. 2005;26(3):302-7.

17. Hoff DN, Dewes H, Rathmann R, Bruch KL, Padula AD. Os desafios da pesquisa e ensino interdisciplinares. Rev Bras Pós-Graduação. 2007;4(7):42-65.

\section{Agradecimentos}

Ao CNPq pelo apoio financeiro e ao Grupo de Pesquisas em Educação em Enfermagem e Saúde - EDEN/UFSC, exemplo concreto da importância do trabalho coletivo a partir da congregação de professores pesquisadores, acadêmicos e profissionais interessados.

Correspondência: Vânia Marli Schubert Backes Rua Victor Konder, 54/303 - Centro CEP 88015-663 - Florianópolis, SC, Brasil 